\title{
An electronmicroscope study of glycopeptide antibiotic- resistant strains of Staphylococcus epidermidis
}

\author{
D. SANYAL* and D. GREENWOOD \\ Department of Microbiology and PHLS Laboratory, University Hospital, Queen's Medical Centre, \\ Nottingham NG7 $5 \mathrm{UH}$
}

\begin{abstract}
Summary. Ultra-thin section transmission electronmicroscopy revealed that two of three glycopeptide-resistant strains of Staphylococcus epidermidis had abnormally thick cell walls, a finding consistent with the view that the reduction in susceptibility may result from the overproduction of glycopeptide binding sites within the cell-wall peptidoglycan. The third resistant strain had a slightly thickened cell wall with an irregular, roughened outline; this strain also underwent autolysis on prolonged incubation on blood agar and the resistance may be associated with abnormal cell-wall synthesis. Sub-MIC concentrations of vancomycin and teicoplanin caused surface damage to a proportion of cocci able to grow in the presence of antibiotic. Exposure to teicoplanin was additionally associated with the formation of filamentous forms and variable amounts of extracellular material. Transmission electronmicroscopy showed that both antibiotics exerted effects within the bacterial cytoplasm of the resistant strains that were not seen in an NCTC control strain: intracellular lamellae and structures resembling mesosomes were observed in the former. These effects were more noticeable in cocci exposed to vancomycin. Bacteria exposed to teicoplanin often showed abnormal septation and, in some preparations, a double-layered cell wall.
\end{abstract}

\section{Introduction}

The glycopeptide antibiotics vancomycin and teicoplanin are active against the majority of gram-positive organisms with the exception of a few insusceptible genera such as Leuconostoc, Pediococcus and Lactobacillus. These antibiotics act by binding to the terminal D-alanyl-D-alanine of the pentapeptide chain of the growing peptidoglycan polymer. ${ }^{1}$ Gramnegative organisms are resistant to these antibiotics by virtue of their outer membrane, through which the relatively large glycopeptide molecule is unable to pass. Vancomycin was developed in $1956^{2}$ and for the next 30 years there were few reports of resistance emerging in gram-positive cocci. This situation has changed since 1986 with the publication of reports documenting glycopeptide resistance in enterococci in several countries. ${ }^{3}$ The biochemical and genetic basis for this form of resistance are now well recognised. ${ }^{4}$ Three resistance phenotypes are described in enterococci. The first, $\operatorname{van} \mathrm{A}$, is the commonest found in this country and is characterised by high-level resistance to both vancomycin and teicoplanin. ${ }^{5}$ It can be transferred to other gram-positive species. ${ }^{6}$ The second,

Received 16 Dec. 1992; accepted 19 Feb. 1993.

* Present address and address for correspondence: Public Health Laboratory, Northern General Hospital, Herries Road, Sheffield S5 7AU. $\operatorname{van} \mathrm{B}$, is characterised by high-level resistance to vancomycin only; susceptibility to teicoplanin is retained. This type of resistance has not been successfully transferred. ${ }^{7}$ The third, van $\mathrm{C}$, is characterised by low-level resistance to vancomycin in some strains of Enterococcus gallinarum and may be an intrinsic property of this species. ${ }^{8}$

The coagulase-negative staphylococci, previously considered to be virtually non-pathogenic, are now commonly encountered in hospital patients, particularly in infections of intravascular catheters, heart valves and cannulae used in continuous ambulatory peritoneal dialysis (CAPD). ${ }^{9}$ Methicillin resistance is common in such isolates and glycopeptide antibiotics are used frequently for treatment. Intraperitoneal vancomycin forms part of the recommended first line of treatment for CAPD infections. ${ }^{10}$

Most reports of coagulase-negative staphylococci with diminished susceptibility to glycopeptide antibiotics describe isolates of Staphylococcus haemolyticus, ${ }^{11-13}$ but glycopeptide-resistant strains of $S$. epidermidis have also been reported. ${ }^{14}$ Isolates of coagulase-negative staphylococci that have been described so far exhibit low-level glycopeptide resistance (MIC $8-32 \mathrm{mg} / \mathrm{L}$ ) and, unlike the situation with enterococci, high-level resistance is, at present, unknown.

In a previous study, the morphological response of susceptible strains of staphylococci to glycopeptides 
was studied by scanning electronmicroscopy. ${ }^{15} \mathrm{We}$ have now investigated three glycopeptide-resistant clinical isolates of $S$. epidermidis and a susceptible type-culture strain. Scanning and transmission electronmicroscopy were used to examine possible ultrastructural differences in the resistant strains and to investigate the response to concentrations of glycopeptides that were below the conventionallydetermined MIC, but within those achievable in plasma on normal dosage.

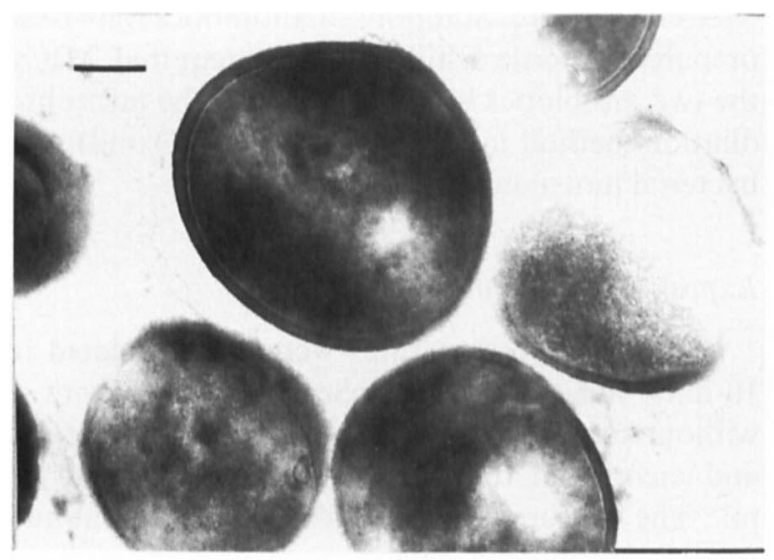

a
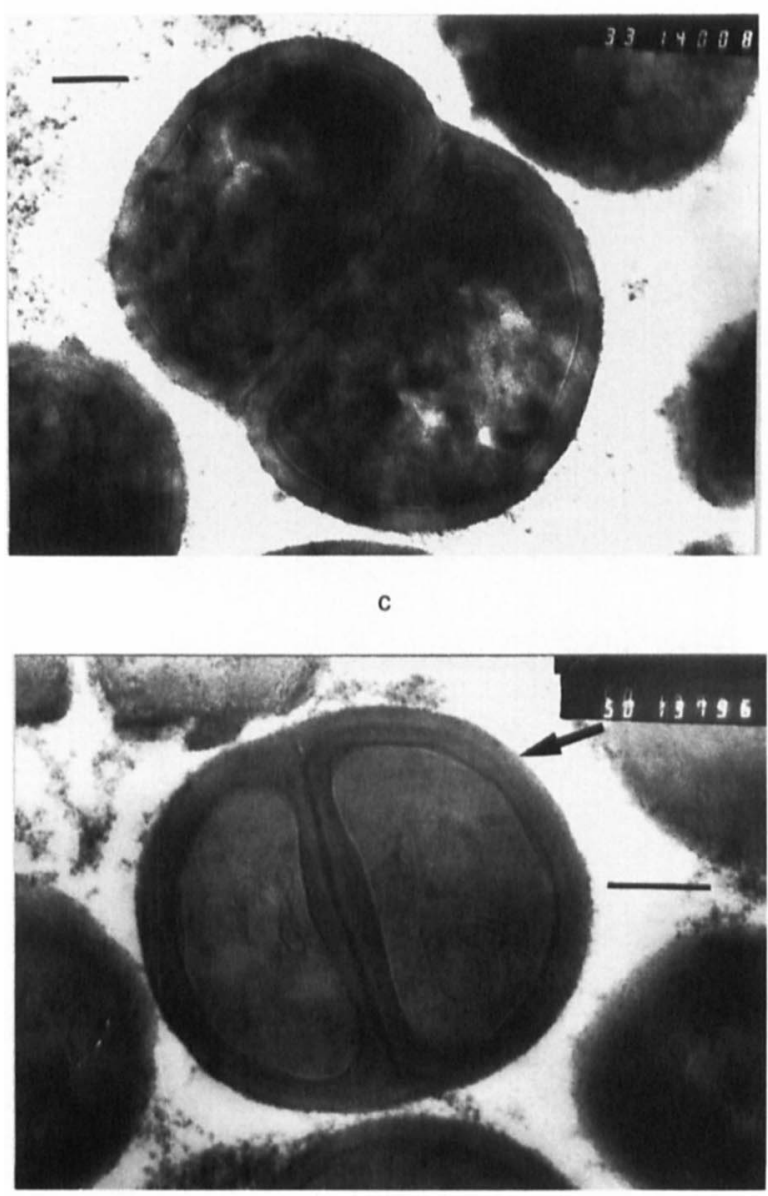

Table. Susceptibilities of the test and control strains to glycopeptide antibiotics determined by broth dilution method

\begin{tabular}{lcrcc} 
& \multicolumn{4}{c}{ MIC (mg/L) of } \\
\cline { 2 - 5 } Glycopeptide epidermidis strains \\
\cline { 2 - 5 } & SP4 & LP4 & JS2 & NCTC 6513 \\
\hline $\begin{array}{l}\text { Vancomycin } \\
\text { Teicoplanin }\end{array}$ & 32 & 8 & 4 & 1 \\
& 32 & 32 & 32 & 0.5 \\
\hline
\end{tabular}
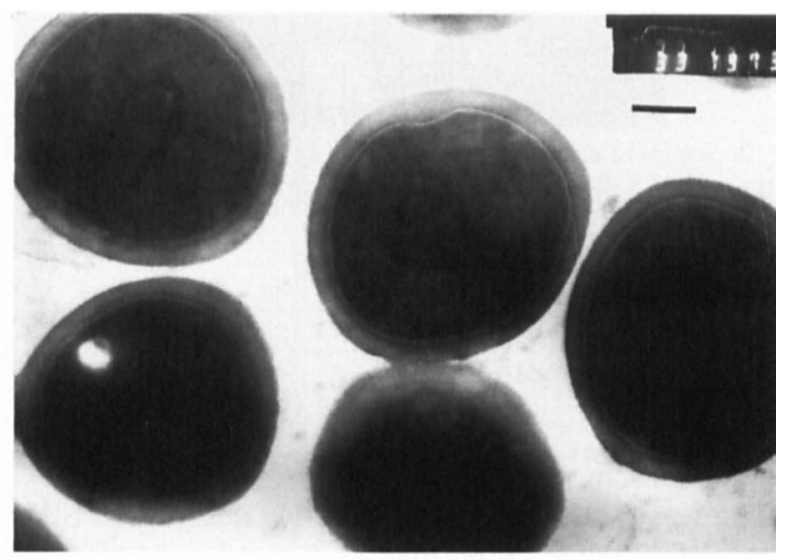

b



d

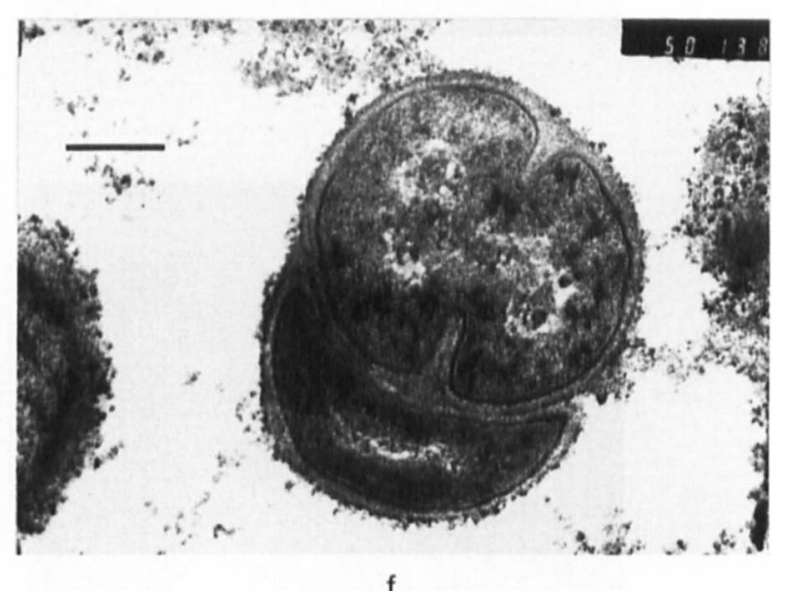

f

Fig. 1. Transmission electronmicroscopy. Ultra-thin sections of the test and control strains stained with uranyl acetate and lead citrate: a, $S$. epidermidis NCTC 6513, b, S. epidermidis SP4 and c, S. epidermidis JS2 grown in the absence of antibiotic; d, S. epidermidis SP4 exposed to vancomycin $8 \mathrm{mg} / \mathrm{L}$ for $18 \mathrm{~h}$ showing intracellular lamellae and mesosomes; e, S. epidermidis SP4 exposed to teicoplanin $16 \mathrm{mg} / \mathrm{L}$ for $18 \mathrm{~h}$ showing double layer $(\rightarrow)$ and mesosomal structures; $f$, S. epidermidis $\mathrm{LP} 4$ exposed to teicoplanin $16 \mathrm{mg} / \mathrm{L}$ for $18 \mathrm{~h}$ showing irregular septation $($ bar $=0.25 \mu \mathrm{m}$ ). 


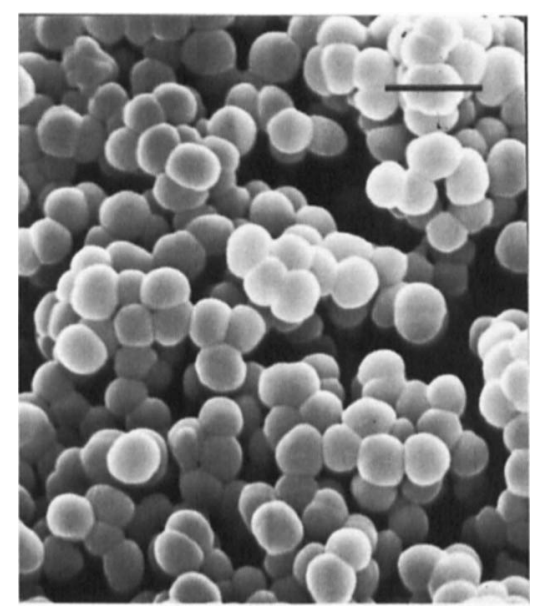

Fig. 2. Scanning electronmicroscopy. S. epidermidis JS2 grown in drug-free broth showing normal appearance of cocci not exposed to antibiotic (bar $=3 \mu \mathrm{m}$ )

\section{Materials and methods}

\section{Bacterial strains}

The test strains of $S$. epidermidis, designated SP4, LP4 and JS2, were identified as $S$. epidermidis by the Staphylococcus Reference Laboratory, Central Public Health Laboratory, Colindale, London. Although

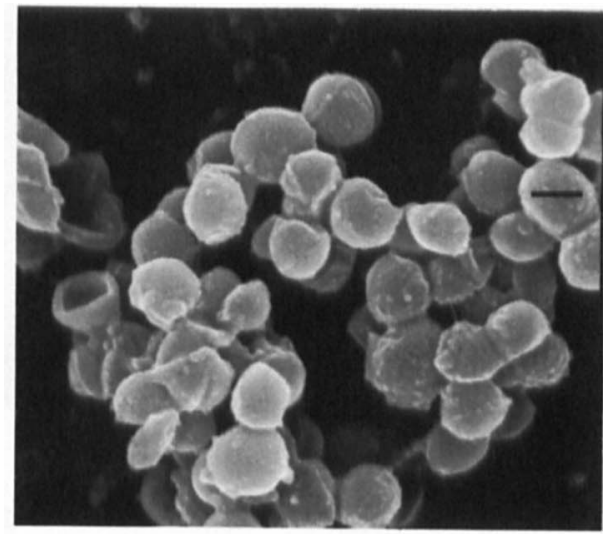

a



c obtained from the same patient, biotyping of strains SP4 and LP4 showed that they were not related. They also differed widely in their antibiotic susceptibility profile and colonial morphology. S. epidermidis NCTC 6513 was used as a glycopeptide-sensitive control.

\section{Antibiotics}

Vancomycin was a standard pharmaceutical preparation for intravenous use obtained from Eli Lilly and Co. Ltd. Teicoplanin was provided by Marion Merrell Dow Ltd. Solutions of antibiotics were freshly prepared in sterile distilled water as required. MICs of the two antibiotics were measured by the macrobroth dilution method in IsoSensitest broth (Oxoid) with a bacterial inoculum of $c .10^{5} \mathrm{cfu} / \mathrm{ml}^{16}$

\section{Exposure to antibiotics}

Overnight broth cultures were subinoculated into $10-\mathrm{ml}$ volumes of fresh IsoSensitest broth, with and without appropriate concentrations of vancomycin and teicoplanin, to achieve an inoculum of $c .10^{5} \mathrm{cfu} /$ $\mathrm{ml}$. The cultures were incubated in a continuous opacity monitoring device based on the simple turbidity cell of Watson et al. ${ }^{17}$ and harvested when growth had reached the stationary phase.

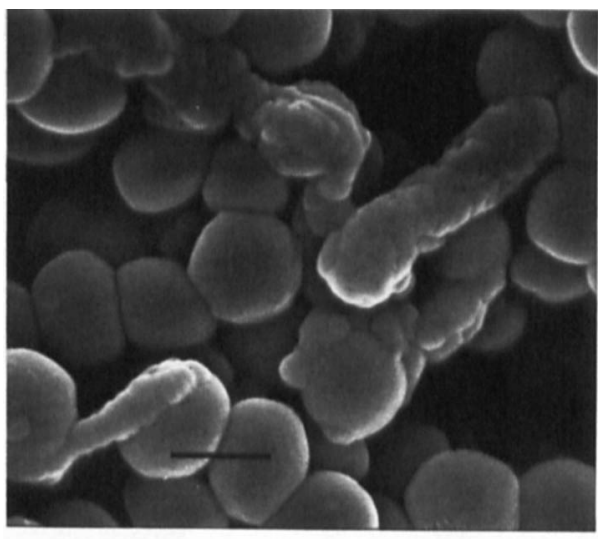

b

Fig. 3. Scanning electronmicroscopy. S. epidermidis NCTC 6513 exposed to: a, vancomycin $0.1 \mathrm{mg} / \mathrm{L}$ for $18 \mathrm{~h} ; \mathbf{b}$ and $\mathbf{c}$, teicoplanin $0.1 \mathrm{mg} / \mathrm{L}$ for $18 \mathrm{~h}($ bar $=1 \mu \mathrm{m})$. 


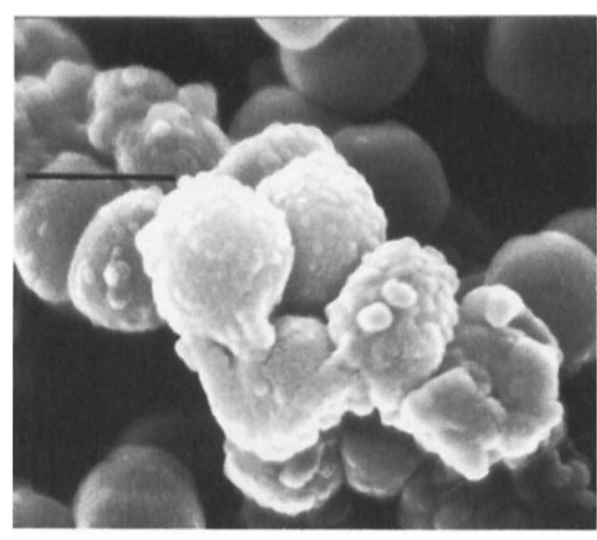

a

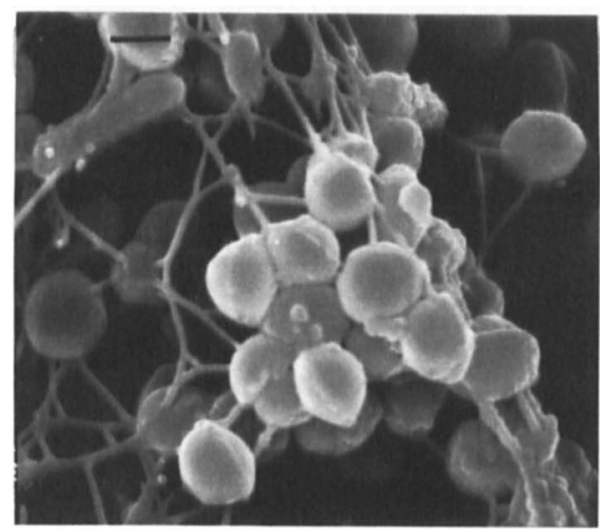

c

\section{Ultra-thin sectioning}

Samples of bacteria were fixed in glutaraldehyde $5 \%$ in $0.05 \mathrm{M}$ cacodylate buffer, $\mathrm{pH} 7.2$, with $10 \mathrm{~mm}$ $\mathrm{MgSO}_{4}$ for $2 \mathrm{~h}$. Samples were embedded by centrifugation ( $2500 \mathrm{rpm}$ for $5 \mathrm{~min}$ ) in agarose $2 \%$ to form a pellet and post-fixed in osmium tetroxide $1 \% \mathrm{w} / \mathrm{v}$ for $16 \mathrm{~h}$ at room temperature. ${ }^{18}$ After three washes in cacodylate buffer, the pellet was cut into $2-3-\mathrm{mm}^{3}$ cubes and dehydrated in a graded series of concentrations of ethanol. The cubes were embedded in a resin mixture of Epon 812 and Araldite CY212 and polymerised at $60^{\circ} \mathrm{C}$ for $24 \mathrm{~h}$ as described by Mollenhauer. ${ }^{19}$ Ultra-thin sections (c. $80 \mathrm{~nm}$ thickness) were cut on an ultramicrotome (Ultratome III, LKB Instruments Ltd) with a freshly-cut glass knife and mounted on formvar-carbon-coated electronmicroscopy grids. The grids were stained with uranyl acetate $(5 \mathrm{~min})$, washed in ethanol $50 \%$ and stained with lead citrate, $\mathrm{pH} 14$, for 5 min. ${ }^{20}$ They were examined in a Jeol $100 \mathrm{C}$ electronmicroscope at an accelerating voltage of $100 \mathrm{kV}$.

To obviate the problem of tangential sectioning, only the thinnest organisms in a field were assessed and photographed. For the purposes of measurement of cell-wall thickness, a minimum of 100 organisms of each strain were photographed. The wall thickness was measured with calibration grids and an average

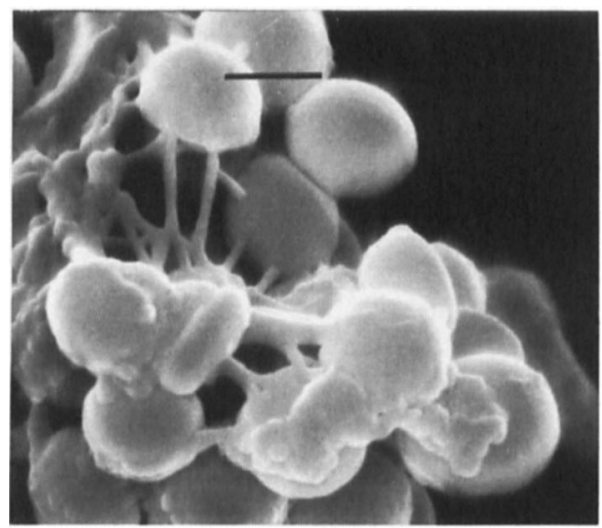

b

Fig. 4. Scanning electronmicroscopy. S. epidermidis SP4 exposed to: a, vancomycin $8 \mathrm{mg} / \mathrm{L}$ for $18 \mathrm{~h}$; b and c, S. epidermidis SP4 exposed to teicoplanin $16 \mathrm{mg} / \mathrm{L}$ for $18 \mathrm{~h}($ bar $=1 \mu \mathrm{m})$.

wall thickness was calculated from the measurements of 100 cells.

\section{Scanning electronmicroscopy}

The method of Elliott and Greenwood ${ }^{21}$ was used. Bacteria harvested in the stationary phase of growth were filtered on to cellulose acetate membranes, fixed with glutaraldehyde $5 \%$ in $0.05 \mathrm{M}$ sodium cacodylate buffer for $1 \mathrm{~h}$, dehydrated in a graded series of concentrations of acetone and critical-point dried in tetramethyl-silane (Sigma). The specimens were then coated with gold in a sputter coater (Balzers Union, Florida USA) and examined with the scanning facility of a Jeol $100 \mathrm{C}$ electronmicroscope at an accelerating voltage of $20 \mathrm{kV}$.

\section{Results}

$M I C s$

The MICs of vancomycin and teicoplanin for the test strains are shown in the table. Turbidimetric results showed that concentrations of each antibiotic of 1 in $2-1$ in 16 MIC had a partial inhibitory effect with subsequent growth during the overnight incubation period, as described previously. ${ }^{22}$ 

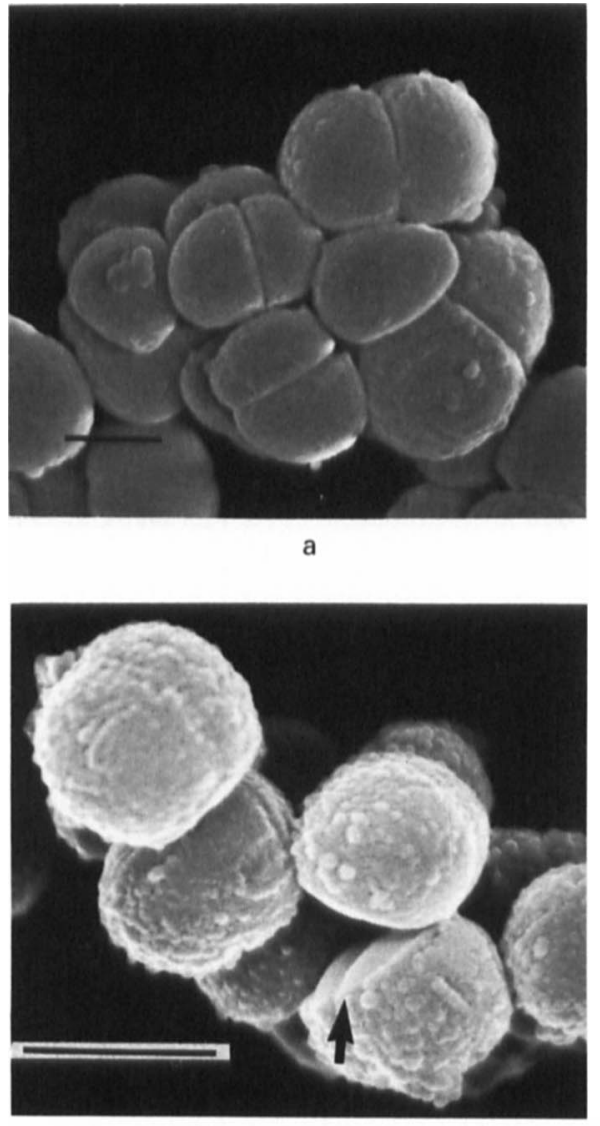

C
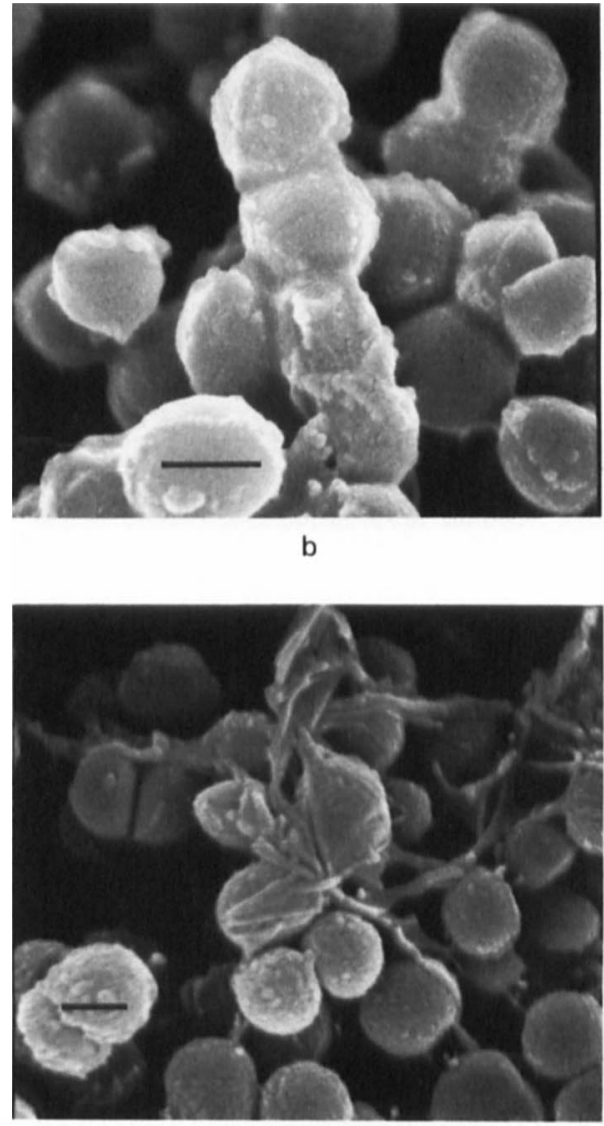

d

Fig. 5. Scanning electronmicroscopy. $S$. epidermidis LP4 exposed to: a, vancomycin $4 \mathrm{mg} / \mathrm{L}$ for $18 \mathrm{~h}$; b, teicoplanin $8 \mathrm{mg} / \mathrm{L}$ for $18 \mathrm{~h}$. $S$. epidermidis JS2 exposed to: c, vancomycin $2 \mathrm{mg} / \mathrm{L}$ for $18 \mathrm{~h}$; d, teicoplanin $8 \mathrm{mg} / \mathrm{L}$ (bar $=1 \mu \mathrm{m}$ ).

\section{Transmission electronmicroscopy}

The cell-wall peptidoglycan layers of $S$. epidermidis SP4 and JS2 were considerably thicker than those of the NCTC control strain even in the absence of antibiotic (fig. 1a-c). The average wall thickness of $S$. epidermidis strains SP4 and JS2 was $60 \mathrm{~nm}$ and $50 \mathrm{~nm}$ respectively, whereas the wall of the NCTC strain had an average thickness of $30 \mathrm{~nm}$. This phenomenon was evident in all cells examined and not just in a proportion of them. The walls of $S$. epidermidis LP4 were only slightly thicker $(c .40 \mathrm{~nm})$ than those of the NCTC strain, but they had a much more irregular outline than those of the other strains. Curiously, colonies of $S$. epidermidis LP4 underwent autolysis when incubated at room temperature on blood agar.

Staphylococci exposed to vancomycin exhibited a variety of cytoplasmic abnormalities including lamellae and mesosome-like structures (fig. 1d). Cells exposed to teicoplanin also showed these structures, but they were less prominent. Cell walls of $S$. epidermidis SP4 exhibited a double layer in the presence of teicoplanin (fig. 1e) and all strains displayed abnormal septation, as shown in fig. If (S. epidermidis LP4).

\section{Scanning electronmicroscopy}

All four strains appeared normal when examined after growth in antibiotic-free broth as shown in fig. 2 (S. epidermidis JS2). After exposure to sub-MIC concentrations of vancomycin, the surface of the cocci of S. epidermidis NCTC 6513 was roughened and blistered, with numerous surface blebs; some of the cocci appeared to have collapsed (fig. 3a). After exposure to similar concentrations of teicoplanin, many of the cocci exhibited gross deformities, sometimes adopting a rod-shaped morphology (fig. 3b), or bearing filamentous extrusions (fig. 3c). Some cells appeared undamaged.

The response of S. epidermidis SP4 to vancomycin at a concentration of $0.5 \mathrm{MIC}$ was similar to that of the NCTC control strain (fig. 4a) with the appearance of bleb-like surface defects scattered over the external cell wall. Sub-MIC concentrations of teicoplanin also caused surface damage, but the preparations were characterised by the presence of large amounts of extracellular material forming a meshwork joining the damaged cells (fig. $4 b$ and c).

The morphological responses to vancomycin and teicoplanin of S. epidermidis LP4 and JS2 (fig. 5a-d) were broadly similar to those seen with other strains, although fibrillar material was less evident after exposure to teicoplanin. A striking finding that was 
particularly evident with $S$. epidermidis JS2 after exposure to vancomycin was an area, apparently associated with points of fission between newly-formed daughter cells, in which the cell wall appeared to have been spared the effects of the antibiotic (shown by the arrow in fig. 5c).

\section{Discussion}

As judged by scanning electronmicroscopy, the surface structure of all four $S$. epidermidis strains appeared completely normal before exposure to antibiotic, but thin-section transmission electronmicroscopy revealed abnormally thick cell walls in the glycopeptide-resistant $S$. epidermidis strains, especially $S$. epidermidis SP4 and JS2. A similar increase in the thickness of the cell wall of $S$. haemolyticus, accompanied by a decline in susceptibility to vancomycin has been reported by Schwalbe et al. ${ }^{23}$ Recently, Daum and co-workers described two strains of $S$. aureus in which isolates trained to grow in increased concentrations of vancomycin had thickened cell walls. ${ }^{24}$

We have presented evidence elsewhere that the resistant strains of $S$. epidermidis examined in the present study bind abnormally large amounts of glycopeptides, especially teicoplanin. ${ }^{22}$ The evident increase in the amount of cell-wall peptidoglycan and the presence of extracellular material observed in S. epidermidis strains SP4 and JS2 after exposure to teicoplanin, provide morphological support for the view that some glycopeptide-resistant strains of coagulase-negative staphylococci possess an excess of glycopeptide binding sites by virtue of overproduction of cell-wall peptidoglycan material. The nature of the extracellular material observed after exposure to teicoplanin remains conjectural.

$S$. epidermidis LP4 was as resistant to teicoplanin as $S$. epidermidis SP4 and JS2, but did not exhibit such thick cell walls. Colonies of this strain on blood agar displayed a marked tendency to autolysis after $48 \mathrm{~h}$ at room temperature, and thin-section electronmicroscopy showed cell walls with an irregular outline. These are unusual characteristics of staphylococci which, being part of the normal skin flora, are well adapted to life outside the body. Cell-wall autolysins

\section{References}

1. Reynolds PE. Structure, biochemistry and mechanism of action of glycopeptide antibiotics. Eur J Clin Microbiol Infect Dis $1989 ; 8$ : 943-950.

2. Griffith RS, Peck FB. Vancomycin, a new antibiotic. III. Preliminary clinical and laboratory studies. In: Welch $\mathrm{H}$ Marti-Ibanez F (eds) Antibiotics annual 1956-1957. New York, Medical Encyclopedia Inc. 1956: 619-622.

3. Johnson AP, Uttley AHC, Woodford N, George RC. Resistance to vancomycin and teicoplanin: an emerging clinical problem. Clin Microbiol Rev 1990; 3: 280-291.

4. Courvalin P. Resistance of enterococci to glycopeptides. Antimicrob Agents Chemother 1990; 34; 2291-2296. are involved in the growth and modification of peptidoglycan and such autolysins in $S$. epidermidis LP4 may also be linked to glycopeptide resistance. Biochemical studies would be needed to substantiate this hypothesis.

The predominant morphological effect of sub-MIC concentrations of vancomycin on $S$. epidermidis NCTC 6513 and the three glycopeptide-resistant strains was similar to that described earlier with supraMIC concentrations of this drug. ${ }^{15}$ The roughened, blistered surfaces of the cocci resembled those of staphylococci exposed to penicillin $\mathrm{s}^{25}$ which also act at the level of the bacterial cell wall. The unusual deformities produced by sub-MIC concentrations of teicoplanin on the NCTC strain were, however, quite different from effects described previously. Similarly, the intracellular lamellae and mesosome-like structures seen by transmission electronmicroscopy do not appear to have been described previously in cocci exposed to glycopeptides. However, a similar phenomenon has been reported in $S$. aureus after treatment with trimethoprim. ${ }^{26}$ In that study, mesosomal bodies and double-layered cell walls similar to those observed in the present investigation were described. Although the primary target of glycopeptides undoubtedly lies in the cell-wall peptidoglycan, it is possible that the secondary consequences of exposure to these agents are felt in other areas of cell metabolism. Indeed, vancomycin may also have an effect on the cell membrane $^{27}$ and RNA synthesis. ${ }^{28}$

Although the clinical isolates examined in the present study were resistant, or of reduced susceptibility, to vancomycin and resistant to teicoplanin according to conventional criteria, the extensive morphological damage observed was achieved with subMIC concentrations of the antibiotics. Such concentrations are readily achievable in therapeutic use and it is possible that sufficient damage may be incurred by the bacteria to make treatment successful. On the other hand, the copious amounts of extracellular material evoked in two of the four strains by exposure to teicoplanin may promote adherence to plastic devices such as CAPD catheters. It would therefore be unwise to draw firm therapeutic conclusions from these findings.

We thank Mr A. P. Shelton for technical advice.

5. Shlaes DM, Bouvet A, Devine C, Shlaes JH, al-Obeid S, Williamson R. Inducible, transferable resistance to vancomycin in Enterococcus faecalis A256. Antimicrob Agents Chemother 1989; 33: 198-203.

6. Leclercq R, Derlot E, Weber M, Duval J, Courvalin P. Transferable vancomycin and teicoplanin resistance in Enterococcus faecium. Antimicrob Agents Chemother 1989; 33: 10-15.

7. Williamson R, al-Obeid S, Shlaes JH, Goldstein FW, Shlaes DM. Inducible resistance to vancomycin in Enterococcus faecium D366. J Infect Dis 1989; 159: 1095-1104.

8. Shlaes DM, Etter L, Gutmann L. Synergistic killing of vancomycin-resistant Enterococci of classes A, B, \& C by combinations of vancomycin, penicillin and gentamicin. Antimicrob Agents Chemother 1991; 35: 776-779. 
9. Archer GL. Antibiotic resistance in coagulase-negative staphylococci. In: Mardh PA, Schleifer KH (eds) Coagulase negative staphylococci. Stockholm, Almquist and Wiksell International. 1986: 93-101.

10. Working Party of the British Society for Antimicrobial Chemotherapy. Diagnosis and management of peritonitis in continuous ambulatory peritoneal dialysis. Lancet 1987 ; 1 : 845-849.

11. Schwalbe RS, Stapleton JT, Gillingan PH. Emergence of vancomycin resistance in coagulase-negative staphylococci. $N$ Engl J Med 1987; 316: 927-931

12. Aubert G, Passot S, Lucht F, Dorche G. Selection of vancomycin- and teicoplanin-resistant Staphylococcus haemolyticus during teicoplanin treatment of $S$. epidermidis infection. J Antimicrob Chemother 1990; 25 : 491-493.

13. Veach LA, Pfaller MA, Barrett M, Koontz FP, Wenzel RP. Vancomycin resistance in Staphylococcus haemolyticus causing colonization and bloodstream infection. $J$ Clin Microbiol 1990; 28: 2064-2068.

14. Sanyal D, Johnson AP, George RC, Cookson BD, Williams AJ. Peritonitis due to vancomycin-resistant Staphylococcus epidermidis. Lancet $1991 ; 337: 54$

15. Greenwood D, Bidgood K, Turner M. A comparison of the responses of staphylococci and streptococci to teicoplanin and vancomycin. J Antimicrob Chemother 1987; 20: 155-164.

16. Waterworth PM. Quantitative methods for bacterial sensitivity testing. In: Reeves DS, Phillips I, Williams JD, Wise R (eds) Laboratory methods in antimicrobial chemotherapy. Edinburgh, Churchill Livingstone. 1978: 31-40.

17. Watson BW, Gauci CL, Blache L, O'Grady F. A simple turbidity cell for continuously monitoring the growth of bacteria. Phys Med Biol 1969; 14: 555-558.

18. Ryter A, Kellenberger E, Birch-Andersen A, Maaloe O. Etude au microscope electronique de plasmas contenant de l'acide desoxyribonucleique. Les nucleosides des bacteries en croissance active. $Z$ Naturforsch $1958 ; 13$ : 597-599.

19. Mollenhauer HH. Plastic embedding mixtures for use in electron microscopy. Stain Technol 1964; 39: 111-115.

20. Reynolds ES. The use of lead citrate at high $\mathrm{pH}$ as an electron opaque stain in electron microscopy. J Cell Biol 1963; 17: 208-213.

21. Elliott TSJ, Greenwood D. The response of Pseudomonas aeruginosa to azlocillin, ticarcillin and cefsulodin. $J \mathrm{Med}$ Microbiol 1983; 16: 351-362.

22. Sanyal D, Johnson AP, George RC, Edwards R, Greenwood D. In-vitro characteristics of glycopeptide resistant strains of Staphylococcus epidermidis isolated from patients on CAPD. J Antimicrob Chemother (in press).

23. Schwalbe RS, Stapleton JT, Gilligan PH. Vancomycin-resistant staphylococcus. $N$ Engl J Med 1987; 317: 767-768.

24. Daum RS, Gupta S, Sabbagh R, Milewski WM. Characterization of Staphylococcus aureus isolates with decreased susceptibility to vancomycin and teicoplanin: isolation and purification of a constitutively produced protein associated with decreased susceptibility. J Infect Dis 1992; 166: 1066-1072.

25. Greenwood D, O'Grady F. Antibiotic-induced surface changes in micro-organisms demonstrated by scanning electron microscopy. Science 1969; 163: 1076-1077.

26. Nishino T, Wecke J, Kruger D, Giesbrecht P. Trimethopriminduced structural alterations in Staphylococcus aureus and the recovery of bacteria in drug-free medium. $J$ Antimicrob Chemother $1987 ; 19: 147-159$.

27. Hancock R, Fitz-James PC. Some differences in the action of penicillin, bacitracin and vancomycin on Bacillus megaterium. J Bacteriol 1964; 87: 1044-1050.

28. Jordan DC, Inniss WE. Selective inhibition of ribonucleic acid synthesis in Staphylococcus aureus by vancomycin. Nature $1959 ; 184$ : 1894-1895. 